\title{
Metabolic Regulation by Nuclear Receptors
}

\author{
Sihao Liu, Michael Downes, and Ronald M. Evans
}

\begin{abstract}
Nuclear receptors (NRs) are a large family of ligand-dependent transcriptional regulators that control development, reproduction, metabolism, and inflammation. Cognate ligands include fatty acids, bile acids, steroids, vitamins A and D, and thyroid hormone, which enable NRs to sense lipophilic nutrients levels and control their respective flux and metabolism. While major metabolic outputs of some individual receptors are well studied, an integrated understanding of their contributions to systemic metabolic homeostasis is just starting to be revealed. In this chapter, we summarize recent advances in NR signaling in metabolism, with a focus on the emerging paradigm that positions NRs as hubs that translate environmental signals of a particular physiological state into daily metabolic rhythms. As NRs are a proven class of therapeutic targets, these novel findings provide insight into therapeutics for the metabolic syndrome.
\end{abstract}

Keywords Nuclear receptors • Energy homeostasis • Metabolite signaling • Microbiome $\bullet$ FGFs $\bullet$ Metabolic diseases

\section{Introduction}

The human body maintains relatively stable levels of key energy substrates through metabolic flexibility [1]. After a meal, elevated blood glucose triggers insulin release to facilitate glucose uptake in the liver and muscles while simultaneously suppressing free fatty acid release from adipose tissue. Between meals, a complex

\footnotetext{
S. Liu $\bullet$ M. Downes

Gene Expression Laboratory, Salk Institute for Biological Studies, 10010 North Torrey Pines Road, La Jolla, CA 92037, USA

R.M. Evans $(\varangle)$

Gene Expression Laboratory, Salk Institute for Biological Studies, 10010 North Torrey Pines Road, La Jolla, CA 92037, USA
}

Howard Hughes Medical Institute, Salk Institute for Biological Studies, 10010 North Torrey Pines Road, La Jolla, CA 92037, USA

e-mail: evans@salk.edu 
counter-regulatory hormonal network maintains a steady blood glucose level via hepatic glycogen breakdown and gluconeogenesis while concurrently decreasing circulating insulin levels to allow adipose tissue lipolysis to release free fatty acids as the major source of energy. Hence, metabolic flexibility can also be described as the ability to switch between carbohydrates and lipids as the predominant source of energy [1].

While metabolic flexibility undoubtedly provided a survival advantage during our early hunter-gatherer times, in combination with excess nutrients it is now driving the human body to a crisis point. Obesity is now a worldwide epidemic and is often accompanied by pathologies such as type 2 diabetes, hepatosteatosis, and cardiovascular diseases. The excessive accumulation of lipids in tissues brought about by modern day excess nutrient uptake, a condition termed as lipotoxicity [2], often results in insulin resistance and hyperglycemia, and leads to metabolic inflexibility of the body.

At the cellular level, metabolic flexibility utilizes energy sensors, among which are nuclear receptors (NRs), that either activate or inhibit specific metabolic pathways. NRs in humans are comprised of a family of 48 ligand-dependent transcription factors with conserved molecular architecture that includes a DNAbinding domain and a ligand-binding domain [3]. Ligands for NRs consist of a diverse array of small lipophilic molecules [3], which, upon binding to their cognate $\mathrm{NR}$, recruit enzymatic cofactors to modulate their target gene expression and, thereby, physiological output. A small subset of NRs have been identified as major players in the maintenance of metabolic flexibility via their ability to sense key lipophilic dietary nutrients and regulate a complex network of genes to control the metabolic flux of these nutrients.

Intensive research over the past two decades has established the fundamental mode of action of a subset of individual receptors (Table 1), including the peroxisome proliferator-activated receptors (PPARs), farnesoid X receptor (FXR), and liver X receptors (LXRs). We will summarize the recent progress in elucidating how these receptors coordinate metabolic homeostasis in response to dietary intake and environmental stressors.

\section{NR Endogenous Ligands Are Dietary Sensors}

The generation and degradation of prototypical endogenous NR ligands through enzymatic modifications, such as those catalyzed by cytochrome P450 (CYP) enzymes [3, 9], allows for integration with NR-independent regulatory pathways. For example, regulation of FOXO1 activity by hepatic insulin signaling determines the expression level of CYP7A1, the rate-limiting P450 enzyme in the synthesis of bile acids, the endogenous ligands for FXR [10].

In the case of the PPARs, each binds fatty acids with relatively low affinity and regulates metabolic homeostasis through canonical direct repeat 1 (DR1) elements within promoters and enhancers of target genes. Specificity is achieved at the level 
Table 1 Nutrient-sensing nuclear receptors

\begin{tabular}{|c|c|c|c|}
\hline & Ligand & Expression & Function \\
\hline $\operatorname{PPAR} \alpha$ & $\begin{array}{l}\text { Short-chain fatty } \\
\text { acids }\end{array}$ & Liver & $\begin{array}{l}\text { PPAR } \alpha \text { was first identified as the } \\
\text { target of the lipid-lowering drug } \\
\text { phenofibrate. PPAR } \alpha \text { is a master } \\
\text { regulator of the adaptive fasting } \\
\text { response in the liver. In the fasted } \\
\text { state, elevated circulating free fatty } \\
\text { acids activate hepatic PPAR } \alpha \text {, } \\
\text { whose targets include rate-limiting } \\
\text { enzymes in fatty acid oxidation and } \\
\text { ketogenesis [4]. PPAR } \alpha \text { knockout } \\
\text { mice die upon prolonged fasting } \\
\text { with excessive hepatic fatty acid } \\
\text { accumulation }\end{array}$ \\
\hline $\operatorname{PPAR} \gamma$ & $\begin{array}{l}\text { 15-deoxy-Delta- } \\
\text { 12,14-prostaglandin } \\
\text { J2 and } \\
\text { thiazolidinedione } \\
\text { antidiabetic drugs }\end{array}$ & Adipose tissue & $\begin{array}{l}\text { PPAR } \gamma \text { is the master regulator of } \\
\text { adipogenesis and lipogenesis, and is } \\
\text { required for both white and brown } \\
\text { adipose tissue formation. } \\
\text { Postprandial increase in fatty acids } \\
\text { activates adipose PPAR } \gamma \text { to facilitate } \\
\text { fat storage and production of } \\
\text { adipokines (leptin, adiponectin, } \\
\text { resistin, etc.), which are important } \\
\text { regulators of whole-body insulin } \\
\text { sensitivity }[4,5]\end{array}$ \\
\hline PPAR $\delta$ & $\begin{array}{l}\text { Long-chain fatty } \\
\text { acids }\end{array}$ & $\begin{array}{l}\text { Ubiquitously } \\
\text { expressed }\end{array}$ & $\begin{array}{l}\text { PPAR } \delta \text { activation enhances fatty } \\
\text { acid oxidation and mitochondrial } \\
\text { respiration. PPAR } \delta \text { activation in } \\
\text { white adipose tissue promotes its } \\
\text { conversion to brown fat, whereas in } \\
\text { the muscle, it increases oxidative } \\
\text { type I fiber formation. In the liver, } \\
\text { PPAR } \delta \text { controls fatty acid synthesis } \\
\text { through direct activation of the } \\
\text { rate-limiting enzyme Acaca }[4,6]\end{array}$ \\
\hline FXR & Bile acids & $\begin{array}{l}\text { Liver, kidney, and } \\
\text { intestine }\end{array}$ & $\begin{array}{l}\text { FXR regulates bile acid homeostasis } \\
\text { by promoting bile acid reabsorption } \\
\text { in the gut and suppressing de novo } \\
\text { bile acid synthesis in the liver }[7,8]\end{array}$ \\
\hline LXR & Oxysterols & $\begin{array}{l}\text { LXR } \alpha \text { : liver, } \\
\text { adipose tissue, and } \\
\text { macrophages; } \\
\text { LXR } \beta \text { : } \\
\text { ubiquitously } \\
\text { expressed }\end{array}$ & $\begin{array}{l}\text { LXRs promote cholesterol efflux in } \\
\text { the liver via } 3 \text { mechanisms: } \\
\text { (1) suppression of cholesterol uptake } \\
\text { from LDL recycling; (2) production } \\
\text { of VLDL for export; (3) conversion } \\
\text { of cholesterol to bile acids for } \\
\text { excretion. LXRs exert an opposing } \\
\text { effect to FXR on bile acid } \\
\text { metabolism [8] }\end{array}$ \\
\hline
\end{tabular}

Acaca acetyl-coA carboxylase $1, F X R$ farnesoid $X$ receptor, $L D L$ low-density lipoprotein, $L X R$ liver $\mathrm{X}$ receptor, $P P A R$ peroxisome proliferator-activated receptor, $V L D L$ very low-density lipoprotein 
of the ligand, enabling the body to enact different physiological responses using common elements within the genome. Long-chain fatty acids preferentially activate PPAR $\delta$ to stimulate fatty acid oxidation in skeletal muscle. In contrast, PPAR $\alpha$ activation by short-chain fatty acids switches on a feed-forward mechanism in the liver to promote fuel oxidation, thereby preventing the intracellular accumulation of fatty acids and consequent lipotoxicity. Thus, the coordinated actions of these PPARs protect against lipotoxicity through the regulation of different metabolic target tissues in response to the uptake of fatty acids.

\section{Gut and Gut Microbiota}

There is growing evidence that the modern lifestyle, most notably the high-fat/highsugar "Western" diet, has altered the genetic composition and metabolic activity of our resident microorganisms. The microbiome communicates with NRs through its ability to modify dietary nutrients to generate specific NR ligands. An early example is oleoylethanolamide (OEA), a high-affinity PPAR $\alpha$ ligand generated in enterocytes from oleic acid [11]. OEA-induced activation of intestinal PPAR $\alpha$ enhances fat absorption through induction of the target gene, CD36, and promotes satiety through a secondary signaling cascade in the brain [12].

More recently, an enhanced bile acid flux was observed throughout the intestinal tract of germ-free mice [13], suggestive of dysregulated FXR activity. While it was known that intestinal microbiota metabolize primary bile acids to increase their hydrophobicity and chemical diversity $[13,14]$, it was not known to what extent the microbiota influence FXR activity. The serum bile acid composition of the germfree mice revealed elevated levels of tauro-conjugated alpha- and beta-muricholic acids (T $\alpha / \beta \mathrm{MCA})$, known FXR antagonists $[13,15]$, which result in a loss of feedback inhibition of bile acid synthesis. Importantly, this study demonstrates that endogenous FXR antagonists are default products of the canonical bile acid synthesis pathway, raising interesting questions about the co-evolution of crucial NR-dependent metabolic pathways and the intestinal microbiome.

An added biological complexity to this system is that reciprocally, bile acids can influence the composition of the intestinal microbiome. A diet high in saturated fatty acids increases tauro-conjugated cholic acid concentrations to promote the growth of sulfate-consuming bacteria, increasing the susceptibility of mice to colitis [16]. This dynamic relationship between bile acids and microbiota is still not well understood, making it difficult to predict the metabolic outcome when deregulation occurs. For example, while FXR null mice are more susceptible to metabolic disorders [17-19], intestinal-specific FXR knockout mice are protected [15]. Clinically, the importance of this pathway was demonstrated in a recent study, which showed that the metabolic benefits of vertical sleeve gastrectomy were mediated by the gut microbiota and FXR signaling in the intestine [20]. 


\section{De Novo Lipogenesis}

In contrast to the relatively well-understood metabolic actions of dietary lipids, the effects of the largely monounsaturated fatty acids generated in the liver through de novo lipogenesis are now being explored [21]. Utilizing genetic mouse models to disrupt hepatic de novo lipogenesis, researchers have demonstrated a broad spectrum of metabolic changes on fat-free diets [22, 23]. Loss of fatty acid synthase (FAS) - a rate-limiting enzyme in de novo lipogenesis - in the liver leads to the development of a fatty liver upon fasting, similar to a PPAR $\alpha$ knockout phenotype $[24,25]$. Importantly, this fatty liver phenotype can be rescued with a PPAR $\alpha$ synthetic ligand, implicating de novo fatty acid synthesis in the generation of endogenous PPAR $\alpha$ ligands. Furthermore, the authors identified a phosphocholine (16:0/18:1-GPC) as an endogenous ligand for PPAR $\alpha$ [24]. In adipose tissue, lipogenesis has been implicated in producing PPAR $\gamma$ endogenous ligands [26], such as 1-O-octadecenyl-2-palmitoyl-3-glycerophosphocholine (18:1e/16:0-GPC) [27].

Direct biochemical approaches have identified endogenous ligands for PPAR $\alpha$ and PPAR $\gamma$. How these ligands function in normal physiology when both dietary and de novo synthesized lipids are present is not clear [28]. Although not directly evaluated under diet-induced or genetic obesity, $\mathrm{PC}(16: 0 / 18: 1)$ is one of two serum phosphocholine species that were observed to have aberrant diurnal rhythmic concentrations [29]. It should be cautioned that "ligands" identified through blocking of an endogenous pathway can be misleading, illustrated by the fact that these phospholipids are weak activators of their respective targets in cell-based reporter systems [24, 27]. However, weak pharmacological activation of PPAR $\alpha$ or PPAR $\gamma$ is beneficial for some metabolic disorders, while, conversely, strong NR activators can carry significant side effects (e.g. rosiglitazone). It was recently shown that a weak PPAR $\gamma$ ligand could achieve similar therapeutic responses to rosiglitazone with limited side effects [30, 31]. Exploring the therapeutic values of these endogenous ligands will be an exciting next step.

\section{Integrative Physiology-Nuclear Receptor-Mediated Crosstalk}

Metabolic flexibility results from cohesive actions of major metabolic organs in response to nutrient flux. It is a collective consequence of localized activation of cellular nutrient-sensing pathways and systemic hormonal actions. Whereas hormones can be released from specialized endocrine organs that directly sense nutrients, they can also be produced from metabolic organs to amplify or propagate local nutrient cues. One example of the latter is the adipose PPAR $\gamma$-mediated production of adipokines such as leptin and adiponectin [5]. In the postprandial setting, 

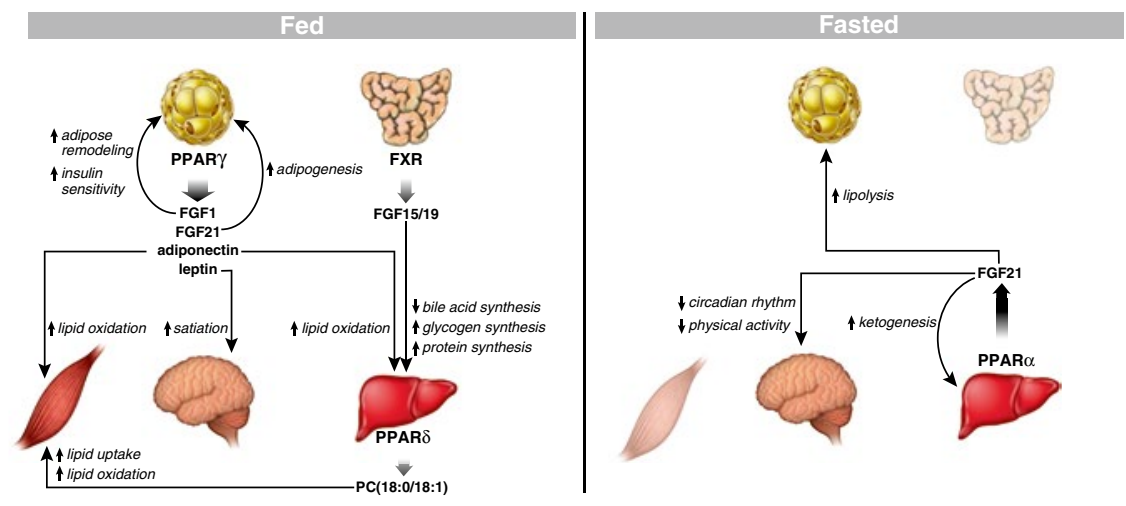

Fig. 1 Integrative physiology through nuclear receptor $(N R)$-mediated crosstalk. Nutrient-sensing NRs amplify or propagate nutrient signals through local and long-range hormone actions. In the fed state, intestinal farnesoid $\mathrm{X}$ receptor $(F X R)$ activation resulting from bile acid release produces fibroblast growth factor $(F G F) 15 / 19$. FGF15/19 limits hepatic bile acid synthesis and promotes glycogen and protein synthesis to store nutrients. Postprandial carbohydrate and lipid influx activate a hepatic peroxisome proliferator-activated receptor $(P P A R) \delta$-controlled lipid synthesis program. The phosphocholine $\mathrm{PC}(18: 0 / 18: 1)$ is secreted as a long-range hormone to promote muscle fat utilization. Feeding activates adipose tissue PPAR $\gamma$, which, in turn, controls the release of multiple adipokines. Locally, FGF21 amplifies PPAR $\gamma$ signaling and promotes further adipogenesis. FGF1 is another paracrine signal that is essential for healthy adipose tissue remodeling and for maintaining whole-body insulin sensitivity. Systemically, adiponectin enhances lipid oxidation in muscle and liver, whereas leptin increases satiation by acting on the central nervous system. During fasting, hepatic PPAR $\alpha$ activation secretes FGF21, whose pleiotropic effects, including elevated adipose tissue lipolysis and hepatic ketogenesis, are important for adaptive fasting response. In addition, FGF21 interferes with the circadian clock in the brain and modulates physical activities

PPAR $\gamma$ induces leptin production to enhance satiation in the central nervous system (CNS), as well as the release of adiponectin to promote fatty acid oxidation in the liver. The dual regulation of central and peripheral responses creates the needed coordination to maintain metabolic balance.

Given the critical roles of the aforementioned NRs in cellular nutrient sensing, it is perhaps not surprising that some of these NRs have been shown to produce hormonelike molecules (Fig. 1). To date, two classes of secreted molecules have been prominently linked with these NRs: the fibroblast growth factors (FGFs) and lipids.

\section{Fibroblast Growth Factors}

Screening for genes activated by nutrient-sensing NRs identified several FGFs as highly induced [32, 33] (Table 2). There are 22 FGFs (FGF1-FGF23) in the human genome (FGF15 is the mouse ortholog of human FGF19 [34]) that are classified as classic or endocrine FGFs. Most classic FGFs are secreted, heparan sulfate-binding 
Table 2 Nutrient-regulated fibroblast growth factors (FGFs)

\begin{tabular}{l|l|l}
\hline Nuclear receptor & FGF & Physiological state \\
\hline FXR & FGF15/19 & Fed \\
\hline PPAR $\alpha$ & FGF21 & Fasted \\
\hline PPAR $\gamma$ & FGF21 & Fed \\
\hline PPAR $\gamma$ & FGF1 & Fed \\
\hline
\end{tabular}

FXR farnesoid $\mathrm{X}$ receptor, $P P A R$ peroxisome proliferatoractivated receptor

proteins that interact locally with fibroblast growth factor receptors (FGFRs) as autocrine/paracrine factors to modulate cell growth, angiogenesis and wound healing [34]. In contrast, the endocrine FGFs (FGF15/19, FGF21, and FGF23) bind heparan sulfate poorly, thereby escaping the extracellular matrix to circulate in the blood. Furthermore, the endocrine FGFs require $\alpha$ - and $\beta$-klotho co-receptors for signal transduction.

After consuming a meal, the liver takes up 25-35\% of dietary carbohydrates [35]. This effect cannot be replicated by either systemic hyperglycemia or hyperinsulinemia, suggesting that crosstalk between the gastrointestinal tract and liver is critical for hepatic glucose utilization. More importantly, feeding-induced hepatic glycogen synthesis remains functional in a genetic model that lacks insulin signaling in the liver $[36,37]$, raising the possibility of a parallel pathway to control postprandial hepatic metabolic reprogramming. Postprandial bile acid release and subsequent reabsorption are integral responses to feeding. The reabsorption of bile acids in the intestine leads to the activation of intestinal FXR and the subsequent secretion of FGF15/19, a direct target of FXR. Originally found to suppress hepatic de novo synthesis of bile acids [32], FGF15/19 has recently been found to promote hepatic glycogen synthesis and protein synthesis by activating the Ras-extracellular signal-related kinase (ERK) pathway, a parallel pathway to the insulin-activated PI3K-AKT pathway [38]. These findings implicate FGF15/19 in the coordination of intestinal feeding responses with hepatic metabolic changes.

In the fasted state, the coordinated activation of hepatic fatty acid oxidation and white adipose tissue lipolysis constitutes a major axis of energy substrate switch from carbohydrate to lipids. FGF21, a hepatic PPAR $\alpha$ target gene, was found to play important roles in liver-adipose crosstalk during fasting [33, 39]. As serum levels of FGF21 correlate closely with hepatic FGF21 expression [40], the liver is thought to be the major source of circulating FGF21. FGF21 promotes free fatty acid release from white adipose tissue by activating several lipases, thereby providing substrates for hepatic fatty acid oxidation and ketogenesis. In addition, FGF21 enhances hepatic ketogenesis through increasing the protein levels of rate-limiting enzymes in ketogenesis.

However, the adaptive fasting response, especially after prolonged fasting, requires the coordinated actions of all major organs to enhance survival. Recently, FGF21 was found to suppress physical activity, alter circadian behavior, and reduce female fertility $[41,42]$ - actions known to be involved in the physiological response to prolonged fasting. Moreover, these adaptive responses were attributed to FGF21 activity in the suprachiasmatic nucleus $(\mathrm{SCN})$ region of the brain. 
Interestingly, pharmacological administration of FGF21 elicits pleiotropic effects beyond the changes seen in the adaptive fasting response [43, 44], suggesting additional roles of FGF21 in other physiological settings. Indeed, FGF21 was found to be a target of PPAR $\gamma$ in adipose tissue, where its paracrine actions potentiate PPAR $\gamma$ activation in the postprandial state [40]. Notably, the effects of FGF21 on glucose homeostasis and hepatic lipid metabolism were shown to be dependent on adiponectin, a PPAR $\gamma$-induced adipokine [44].

In addition to liver and white adipose tissue, FGF21 is produced in brown fat [45] and skeletal muscle [46] in response to specific stimuli, suggesting that FGF21 coordinates the tissue-specific responses of the major metabolic organs.

The expressions of FGF15/19 and FGF21 are exquisitely dynamic during fasting and feeding cycles [32, 33, 40, 47]. The nutrient-responsive nature of FGF21 and FGF15 expression opens a new chapter for their respective roles in metabolism. In contrast, the prototypic FGF1 and FGF2 [34] are ubiquitously expressed [48], with established roles in wound healing and brain development [34]. Whereas FGF2 knockout mice have mild brain development and wound healing defects, FGF1 knockout mice are phenotypically normal [49]. In search of additional FGFs that may act as nutrient-controlled hormones, FGF1 was found to be highly induced in white adipose tissue upon feeding of a high-fat diet [50]. Conversely, FGF1 knockout (FGF1KO) mice developed marked adipose tissue fibrosis, impaired glucose homeostasis, and insulin insensitivity when fed a high-fat diet. Furthermore, upon withdrawal of the high-fat diet, FGF1KO mice developed pronounced fat necrosis. Given that adipose tissue expansion accompanied by increased vascularization is required to maintain insulin sensitivity under conditions of nutrient surplus [51], the poor white adipose tissue vascularization observed in FGF1 knockout mice may be responsible for their severe insulin-resistant phenotype on a high-fat diet. Interestingly, FGF1 is shown to be directly regulated by PPAR $\gamma$, and PPAR $\gamma$ and its target genes are increased in FGF1 knockout mice [50]. Therefore, FGF1 appears to be an essential output for PPAR $\gamma$-mediated insulin sensitization. Distinct from FGF21-mediated amplification of PPAR $\gamma$-mediated adipogenesis and adipokine production [40], FGF1 does not promote adipogenesis in 3 T3-L1 cells [52]. These properties raise the intriguing possibility of recombinant FGF1 (rFGF1) as an antidiabetic drug that may minimize the side effects associated with the thiazolidinedione class of PPAR $\gamma$ agonists. Indeed, rFGF1 injection markedly improves insulin sensitivity in genetic and diet-induced obesity models [53]. These effects are insulin dependent, suggesting a role as an insulin sensitizer, and require adipose FGFR1 expression, as rFGF1 fails to lower blood glucose in FGFR1 adiposespecific knockout mice. Interestingly, $\mathrm{rFGF} 1$ treatment does not alter adipose tissue vasculature, indicating differences between pharmacological administration and genetic studies. Importantly, an N-terminally truncated FGF1 analog with markedly reduced mitogenic activity was similarly able to normalize blood glucose levels in 
diabetic mice [53]. The ability to dissociate the mitogenic and glucose-lowering activities of FGF1 suggests that these different effects may be receptor specific. Nevertheless, these results demonstrate that FGF1 is a critical mediator of nutrient responsiveness in adipose tissue and has significant therapeutic potential.

\section{Lipids}

De novo lipogenesis has emerged as a novel pathway that produces lipid signaling molecules, including NR ligands. It was found that activation of PPAR $\delta$ in the liver enhanced hepatic de novo lipogenesis but paradoxically reduced serum lipid concentrations $[6,29]$. The reduction of serum lipids was also correlated with increased fatty acid uptake and oxidation in the skeletal muscle. Through unbiased metabolomics profiling, phosphocholine $\mathrm{PC}(18: 0 / 18: 1)$ was identified as a signaling molecule that facilitates postprandial crosstalk between the liver and muscle to promote fatty acid utilization. Furthermore, the effects of PC(18:0/18:1) are mediated by another NR, PPAR $\alpha$, in the muscle, underlying a new model of tissue crosstalk through NRs.

\section{Concluding Remarks}

In 1995, 10 years after the cloning of the first NR, the glucocorticoid receptor, the challenge of the field was proposed: "...to elucidate the contributions of these individual systems to the integrated and complex processes associated with the multihormonal nature of metabolic regulation..." [54] This review summarizes our current understandings toward this challenge. An immediate implication of our expanded knowledge of endogenous ligands and downstream hormonal signals is to explore their potential as novel therapeutics. Indeed, several compounds are in clinical trials for metabolic syndrome. In addition, these recent advances have highlighted the role of nutrient-sensing NRs in coordinating metabolic homeostasis. Through receptor activation, environmental cues are transduced as local metabolic outputs and long-range hormonal actions (Fig. 2). In a reductionist's perspective, these receptors may be important on-off switches for specific physiological states [55]. While wholesale, prolonged activation of any of these receptors may not be desirable, the use of high-affinity synthetic ligands offers a window to tease out beneficial downstream targets, especially in light of the identification of several hormone-like molecules that are good therapeutic candidates. With readily available synthetic ligands and mouse genetic models, it is possible that these receptors will become platforms for drug discovery. 

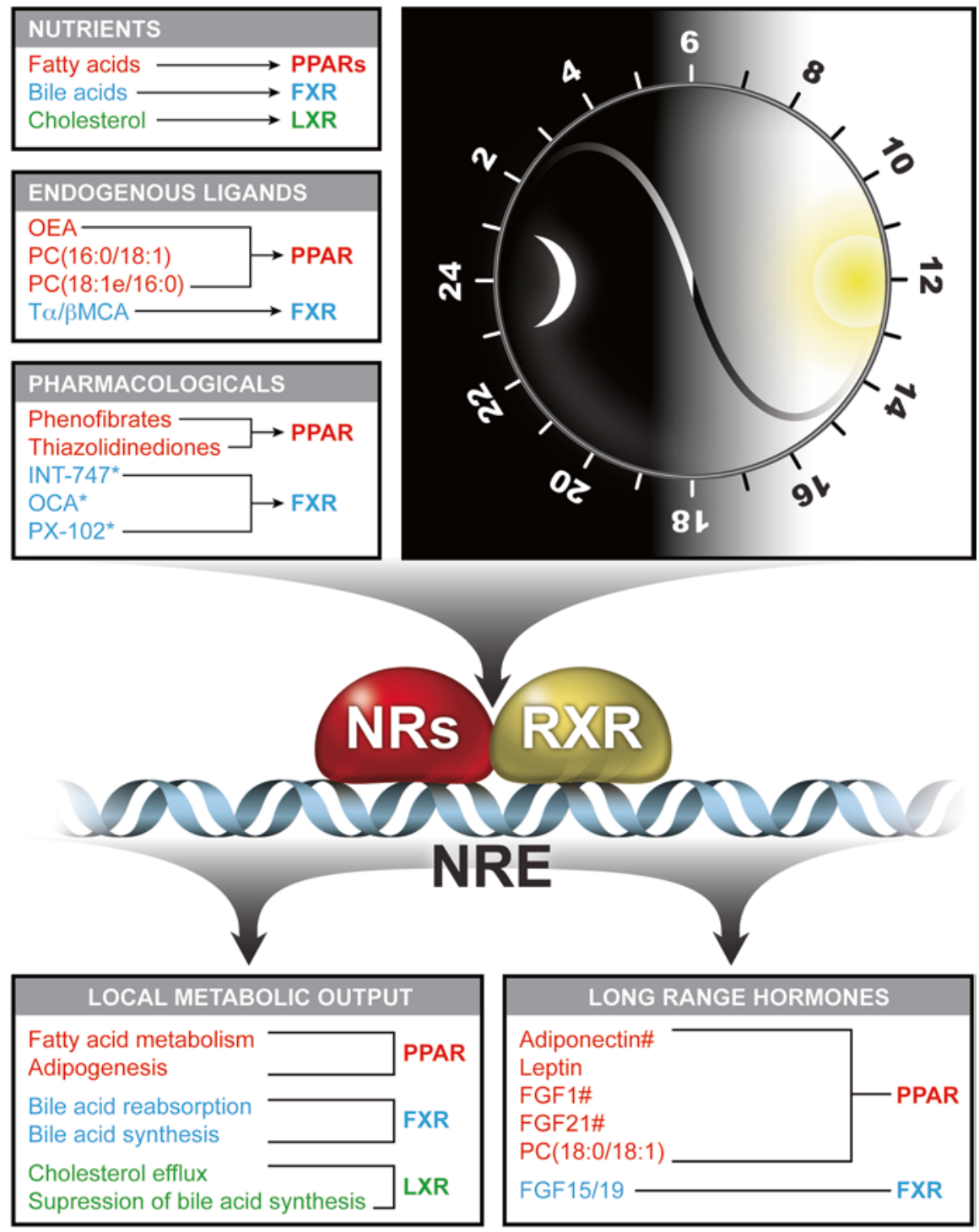

*currently under clinical trial \#pharmacological target

Fig. 2 Nuclear receptors $(N R s)$ as hubs to transduce environmental signals into daily metabolic rhythms. Originally identified as sensors for lipophilic nutrients to control their respective ligand metabolism, emerging evidence points to an integrative nature of the regulation and action of the peroxisome proliferator-activated receptor $(P P A R)$, farnesoid $\mathrm{X}$ receptor $(F X R)$, and liver $\mathrm{X}$ receptor $(L X R)$ families of NRs. In addition to nutrients, their activities are fine-tuned by endogenous ligands and the circadian clock. Activation of these NRs not only controls key cellular metabolic programs but also produces hormones that further connect metabolic tissues for a cohesive response to nutrient flux. Given the central roles of NRs in nutrient signaling, several pharmacological agents have been developed or are in development for metabolic diseases. Additionally, the NR-controlled hormones represent yet another emerging venue for therapeutics 
Open Access This chapter is distributed under the terms of the Creative Commons Attribution Noncommercial License, which permits any noncommercial use, distribution, and reproduction in any medium, provided the original author(s) and source are credited.

\section{References}

1. Muoio DM, Newgard CB (2006) Obesity-related derangements in metabolic regulation. Annu Rev Biochem 75:367-401

2. Samuel VT, Shulman GI (2012) Mechanisms for insulin resistance: common threads and missing links. Cell 148(5):852-871

3. Evans RM, Mangelsdorf DJ (2014) Nuclear receptors, RXR, and the big bang. Cell 157(1):255-266

4. Evans RM, Barish GD, Wang YX (2004) PPARs and the complex journey to obesity. Nat Med 10(4):355-361

5. Ahmadian $\mathrm{M}$ et al (2013) PPARgamma signaling and metabolism: the good, the bad and the future. Nat Med 19(5):557-566

6. Liu S et al (2011) Role of peroxisome proliferator-activated receptor $\{$ delta $\} /\{$ beta $\}$ in hepatic metabolic regulation. J Biol Chem 286(2):1237-1247

7. de Aguiar Vallim TQ, Tarling EJ, Edwards PA (2013) Pleiotropic roles of bile acids in metabolism. Cell Metab 17(5):657-669

8. Calkin AC, Tontonoz P (2012) Transcriptional integration of metabolism by the nuclear sterolactivated receptors LXR and FXR. Nat Rev Mol Cell Biol 13(4):213-224

9. Chawla A et al (2001) Nuclear receptors and lipid physiology: opening the X-files. Science 294(5548): 1866-1870

10. Haeusler RA et al (2012) Impaired generation of 12-hydroxylated bile acids links hepatic insulin signaling with dyslipidemia. Cell Metab 15(1):65-74

11. Fu J et al (2003) Oleylethanolamide regulates feeding and body weight through activation of the nuclear receptor PPAR-alpha. Nature 425(6953):90-93

12. Gaetani $\mathrm{S}$ et al (2010) The fat-induced satiety factor oleoylethanolamide suppresses feeding through central release of oxytocin. J Neurosci 30(24):8096-8101

13. Sayin SI et al (2013) Gut microbiota regulates bile acid metabolism by reducing the levels of tauro-beta-muricholic acid, a naturally occurring FXR antagonist. Cell Metab 17(2):225-235

14. Swann JR et al (2011) Systemic gut microbial modulation of bile acid metabolism in host tissue compartments. Proc Natl Acad Sci U S A 108(Suppl 1):4523-4530

15. Li F et al (2013) Microbiome remodelling leads to inhibition of intestinal farnesoid X receptor signalling and decreased obesity. Nat Commun 4:2384

16. Devkota $S$ et al (2012) Dietary-fat-induced taurocholic acid promotes pathobiont expansion and colitis in Il10-/- mice. Nature 487(7405):104-108

17. Cariou $B$ et al (2006) The farnesoid $X$ receptor modulates adiposity and peripheral insulin sensitivity in mice. J Biol Chem 281(16):11039-11049

18. Ma K et al (2006) Farnesoid X receptor is essential for normal glucose homeostasis. J Clin Invest 116(4):1102-1109

19. Zhang Y et al (2006) Activation of the nuclear receptor FXR improves hyperglycemia and hyperlipidemia in diabetic mice. Proc Natl Acad Sci U S A 103(4):1006-1011

20. Ryan KK et al (2014) FXR is a molecular target for the effects of vertical sleeve gastrectomy. Nature 509(7499):183-188

21. Fu S et al (2011) Aberrant lipid metabolism disrupts calcium homeostasis causing liver endoplasmic reticulum stress in obesity. Nature 473(7348):528-531

22. Liu S, Alexander RK, Lee CH (2014) Lipid metabolites as metabolic messengers in interorgan communication. Trends Endocrinol Metab 25(7):356-363 
23. Lodhi IJ, Wei X, Semenkovich CF (2011) Lipoexpediency: de novo lipogenesis as a metabolic signal transmitter. Trends Endocrinol Metab 22(1):1-8

24. Chakravarthy MV et al (2009) Identification of a physiologically relevant endogenous ligand for PPARalpha in liver. Cell 138(3):476-488

25. Chakravarthy MV et al (2005) "New" hepatic fat activates PPARalpha to maintain glucose, lipid, and cholesterol homeostasis. Cell Metab 1(5):309-322

26. Kim JB, Spiegelman BM (1996) ADD1/SREBP1 promotes adipocyte differentiation and gene expression linked to fatty acid metabolism. Genes Dev 10(9):1096-1107

27. Lodhi IJ et al (2012) Inhibiting adipose tissue lipogenesis reprograms thermogenesis and PPARgamma activation to decrease diet-induced obesity. Cell Metab 16(2):189-201

28. Kim YG, Lou AC, Saghatelian A (2011) A metabolomics strategy for detecting protein-metabolite interactions to identify natural nuclear receptor ligands. Mol Biosyst 7(4):1046-1049

29. Liu $\mathrm{S}$ et al (2013) A diurnal serum lipid integrates hepatic lipogenesis and peripheral fatty acid use. Nature 502(7472):550-554

30. Choi JH et al (2010) Anti-diabetic drugs inhibit obesity-linked phosphorylation of PPARgamma by Cdk5. Nature 466(7305):451-456

31. Choi JH et al (2011) Antidiabetic actions of a non-agonist PPARgamma ligand blocking Cdk5mediated phosphorylation. Nature 477(7365):477-481

32. Inagaki $\mathrm{T}$ et al (2005) Fibroblast growth factor 15 functions as an enterohepatic signal to regulate bile acid homeostasis. Cell Metab 2(4):217-225

33. Inagaki $T$ et al (2007) Endocrine regulation of the fasting response by PPARalpha-mediated induction of fibroblast growth factor 21. Cell Metab 5(6):415-425

34. Beenken A, Mohammadi M (2009) The FGF family: biology, pathophysiology and therapy. Nat Rev Drug Discov 8(3):235-253

35. Moore MC et al (2012) Regulation of hepatic glucose uptake and storage in vivo. Adv Nutr 3(3):286-294

36. Dong XC et al (2008) Inactivation of hepatic Foxo1 by insulin signaling is required for adaptive nutrient homeostasis and endocrine growth regulation. Cell Metab 8(1):65-76

37. Lu M et al (2012) Insulin regulates liver metabolism in vivo in the absence of hepatic Akt and Foxo1. Nat Med 18(3):388-395

38. Kir S et al (2011) FGF19 as a postprandial, insulin-independent activator of hepatic protein and glycogen synthesis. Science 331(6024):1621-1624

39. Badman MK et al (2007) Hepatic fibroblast growth factor 21 is regulated by PPARalpha and is a key mediator of hepatic lipid metabolism in ketotic states. Cell Metab 5(6):426-437

40. Dutchak PA et al (2012) Fibroblast growth factor-21 regulates PPARgamma activity and the antidiabetic actions of thiazolidinediones. Cell 148(3):556-567

41. Bookout AL et al (2013) FGF21 regulates metabolism and circadian behavior by acting on the nervous system. Nat Med 19(9):1147-1152

42. Owen BM et al (2013) FGF21 contributes to neuroendocrine control of female reproduction. Nat Med 19(9):1153-1156

43. Xu J et al (2009) Fibroblast growth factor 21 reverses hepatic steatosis, increases energy expenditure, and improves insulin sensitivity in diet-induced obese mice. Diabetes 58(1):250-259

44. Lin Z et al (2013) Adiponectin mediates the metabolic effects of FGF21 on glucose homeostasis and insulin sensitivity in mice. Cell Metab 17(5):779-789

45. Hondares E et al (2011) Thermogenic activation induces FGF21 expression and release in brown adipose tissue. J Biol Chem 286(15):12983-12990

46. Kim KH et al (2013) Autophagy deficiency leads to protection from obesity and insulin resistance by inducing Fgf21 as a mitokine. Nat Med 19(1):83-92

47. Stroeve JH et al (2010) Intestinal FXR-mediated FGF15 production contributes to diurnal control of hepatic bile acid synthesis in mice. Lab Invest 90(10):1457-1467

48. Fon Tacer $\mathrm{K}$ et al (2010) Research resource: comprehensive expression atlas of the fibroblast growth factor system in adult mouse. Mol Endocrinol 24(10):2050-2064 
49. Miller DL et al (2000) Compensation by fibroblast growth factor 1 (FGF1) does not account for the mild phenotypic defects observed in FGF2 null mice. Mol Cell Biol 20(6):2260-2268

50. Jonker JW et al (2012) A PPARgamma-FGF1 axis is required for adaptive adipose remodelling and metabolic homeostasis. Nature 485(7398):391-394

51. Sun K, Kusminski CM, Scherer PE (2011) Adipose tissue remodeling and obesity. J Clin Invest 121(6):2094-2101

52. Hutley L et al (2004) Fibroblast growth factor 1: a key regulator of human adipogenesis. Diabetes 53(12):3097-3106

53. Suh JM et al (2014) Endocrinization of FGF1 produces a neomorphic and potent insulin sensitizer. Nature 513(7518):436-439

54. Mangelsdorf DJ, Evans RM (1995) The RXR heterodimers and orphan receptors. Cell 83(6):841-850

55. Fan W et al (2013) Road to exercise mimetics: targeting nuclear receptors in skeletal muscle. J Mol Endocrinol 51(3):T87-T100 